\title{
Comment on 'increased frequency of combined methylenetetrahydrofolate reductase C677T and A1298C mutated alleles in spontaneously aborted embryos'
}

\author{
European Journal of Human Genetics (2002) 10, 578-579. doi:10.1038/sj.ejhg.5200862
}

We read with great interest the recent article by Zetterberg et $a l .{ }^{1}$ that examined the potential role of methylenetetrahydrofolate reductase (MTHFR) gene polymorphisms in contributing to early spontaneous abortions. Zetterberg et al. ${ }^{1}$ determined the incidence of C677T and A1298C MTHFR mutations in a study cohort $(n=80)$, which consisted of foetal tissue samples from early spontaneous abortions, and a control group $(n=125)$, which consisted of DNA samples from healthy blood donors that were presumably chosen at random. In their study group, early spontaneous abortions were defined as foetal deaths that occurred before the twentieth week of gestation. The earliest foetal death occurred at 6 weeks gestation and the majority, $87.5 \%$ of foetal deaths, occurred before the twelfth week of gestation. Both study and control groups were from the island of Crete. Using a solid-phase minisequencing technique, Zetterberg et al. ${ }^{1}$ determined that combined 677TT/1298AA and 677CT/ 1298AC genotypes were observed more frequently in their foetal study group than in their adult control group (Odds ratio [OR] of 2.06, $P=0.015)$. They also observed that MTHFR mutations, taking into account single or multiple mutations, were more common in the foetal group than adult controls (OR of 14.2, $P=0.001$ ), suggesting to them that MTHFR mutations may play a significant role in determining foetal viability, especially during times of high folate demand. Zetterberg et al. ${ }^{1}$ only observed MTHFR mutations in trans positions and did not identify any 677TT/1298AC, 677CT/1298CC or C77TT/1298CC genotype combinations in either their study or control groups. They propose that there is complete linkage disequilibrium between the two common MTHFR polymorphisms, C677T and A1298C, as MTHFR mutations in cis positions were not identified in their study.

Complete linkage disequilibrium between the C677T and A1298C mutations has been previously proposed, ${ }^{2-4}$ however, there is substantial evidence indicating that any linkage disequilibrium between MTHFR mutations is likely incomplete. $^{5-11}$ The discovery of MTHFR mutations in cis positions is of great importance, as it allows multiple, specifically triple and quadruple MTHFR mutations, to occur within a genome. These compound MTHFR mutations may have deleterious clinical effects, especially during times of folate insufficiency.

We have previously demonstrated the existence of all MTHFR C677T and A1298C MTHFR genotype combinations in a foetal study group. ${ }^{8}$ The absence of $677 \mathrm{CT} / 1298 \mathrm{CC}$ and 677TT/1298CC genotype combinations in our neonatal control group ${ }^{8}$ provides strong evidence that compound triple and quadruple MTHFR mutations may be associated with compromised foetal viability. There has been some criticism with the MboII restriction fragment length polymorphism detection of $\mathrm{A} 1298 \mathrm{C}$ mutations, due to possible interference from silent T1317C mutations ${ }^{5}$ however, these criticisms are unfounded, especially since the identification of mutated alleles would not be compromised. ${ }^{12}$ A recent study by Volcik et al. ${ }^{11}$ has even demonstrated the existence of a MTHFR 677TT/1298CC genotype in a living Hispanic woman who had a child with spina bifida, providing further evidence that recombinant events between MTHFR mutation loci do occur despite their short physical distance of $2.1 \mathrm{~kb}{ }^{8}$

In an adult Canadian population of predominantly European (Celtic) descent and other ethnic backgrounds, we have demonstrated a relatively high frequency of 677TT/1298AC genotypes, ranging from 9.2 to $12.5 \%$ in study and control groups, respectively, indicating a strong incidence of cis MTHFR mutations in this population. ${ }^{9,13}$ This is in contrast to Weisberg et al. ${ }^{5}$ who studied Canadian groups of 133 children with spina bifida and 141 mothers of children with spina bifida and discovered only a single child with a MTHFR 677TT/1298AC genotype. Although Canadian, the ethnic heritage of their study population is unknown.

The seemingly conflicting reports regarding the identification of cis and trans MTHFR mutations are probably best explained by the heterogeneity of the study populations. There is no doubt that there are both ethnic and regional differences in MTHFR C677T allele distributions, as well as A1298C allele distributions. ${ }^{1-4,6,8,9,11}$ The allele frequency of MTHFR C677T mutations ranges dramatically from 0.052 in African Blacks to 0.487 in Columbians. ${ }^{3}$ Even within a country, such as Canada, there are regional differences in MTHFR allele distributions, as the Canadian population is ethnically heterogeneous. Canadian MTHFR 
677T allele frequencies have been reported in Canadian Inuit $(6.1 \%),{ }^{14}$ Manitoba neonates $(24.97 \%),{ }^{15}$ French Canadians $(38 \%)^{16}$ and our neonatal $(27.3 \%)^{8}$ and adult (40\%) control and study groups. ${ }^{9}$ Considering the regional variations in MTHFR allele distributions, it is not completely surprising that some studies have recognised the common MTHFR mutations only in trans positions. ${ }^{1-4}$ It is quite possible that in certain populations, such as on the island of Crete recombinant events between MTHFR $677 \mathrm{~T}$ and $1298 \mathrm{C}$ mutations are very rare occurrences and identification of any cis mutations would require genotyping of a large number of individuals.

Although Zetterberg et al. ${ }^{1}$ investigated early spontaneous abortions, with all foetal deaths occurring before 6 and 20 weeks of gestation, the inherent limitation of their study, as well as other studies investigating foetal viability, ${ }^{8}$ is that very early spontaneous abortions are not examined in study populations. The true contribution of MTHFR mutations to reduced foetal viability may be difficult to determine, as the majority of pregnancies that spontaneously terminate before 6 weeks of gestation is not identified clinically. It is evident that additional large-scale population studies are required to fully elucidate the role of MTHFR mutations in contributing to decreased foetal viability. It also appears that regionally based studies are necessary to assess risks for specific populations.

Phillip A Isotalo

Department of Pathology and Laboratory Medicine, Mayo Clinic, Rochester, Minnesota, USA James G Donnelly Department of Pathology, New York University School of Medicine, New York, New York, USA

\section{References}

1 Zetterberg H, Regland B, Palmer $M$ et al: Increased frequency of combined methylenetetrahydrofolate reductase C677T and A1298C mutated alleles in spontaneously aborted embryos. Eur J Hum Genet 2002; 10: 113-118.

2 van der Put NMJ, Gabreels F, Stevens EMB et al: A second common mutation in the methylenetetrahydrofolate reductase gene: an additional risk factor for neural-tube defects?. Am J Hum Genet 1998; 62: 1044-1051.
3 Rady PL, Tyring SK, Hudnall SD et al: Methylenetetrahydrofolate reductase (MTHFR): the incidence of mutations C677T and A1298C in the Ashkenazi Jewish population. Am J Med Genet 1999; 86: 380-384.

4 Stegmann K, Ziegler A, Ngo ET et al: Linkage disequilibrium of MTHFR genotypes 677C/T-1298A/C in the German population and association studies with neural tube defects (NTD). Am J Med Genet 1999; 87: 23-29.

5 Weisberg I, Tran P, Christensen B, Sibani S, Rozen R: A second genetic polymorphism in methylenetetrahydrofolate reductase (MTHFR) associated with decreased enzyme activity. Mol Genet Metab 1998; 64: 169-172.

6 Friedman G, Goldschmidt N, Friedlander Y et al: A common mutation $\mathrm{A} 1298 \mathrm{C}$ in human methylenetetrahydrofolate reductase gene: association with plasma total homocysteine and folate concentrations. J Nutr 1999; 129: 1656-1661.

7 Skibola CF, Smith MT, Kane E et al: Polymorphisms in the methylenetetrahydrofolate reductase gene are associated with susceptibility to acute leukemia in adults. Proc Natl Acad Sci USA 1999; 96: 12810-12815.

8 Isotalo PA, Wells GA, Donnelly JG: Neonatal and fetal methylenetetrahydrofolate reductase genetic polymorphisms: an examination of C677T and A1298C mutations. Am J Hum Genet 2000; 67: 986-990.

9 Isotalo PA, Donnelly JG: Prevalence of methylenetetrahydrofolate reductase mutations in patients with venous thrombosis. Mol Diagn 2000; 5: 59-66.

10 Hanson NQ, Aras O, Yang F, Tsai MY: C677T and A1298C polymorphisms of the methylenetetrahydrofolate reductase gene: incidence and effect of combined genotypes on plasma fasting and post-methionine load homocysteine in vascular disease. Clin Chem 2001; 47: 661-667.

11 Volcik KA, Blanton SH, Northrup H: Examinations of methylenetetrahydrofolate reductase C677T and A1298C mutations - and in utero viability [Letter]. Am J Hum Genet 2001; 69: 1150-1152.

12 Donnelly JG: The silent T1317C mutation of methylenetetrahydrofolate reductase should not interfere with MboII restriction isotyping of the reported A1298C mutation. Mol Genet Metab 1999; 68: 511.

13 Donnelly JG, Isotalo PA: Reply to 'Examinations of methylenetetrahydrofolate reductase C677T and A1298C mutations - and in utero viability' [Letter]. Am J Hum Genet 2001; 69: 1152-1153.

14 Hegele RA, Tully C, Young TK, Connelly PW: V677 mutation of methylenetetrahydrofolate reductase and cardiovascular disease in Canadian Inuit [Letter]. Lancet 1997; 349: 1221-1222.

15 Mogk RL, Rothenmund H, Evans JA, Carson N, Dawson AJ: The frequency of the C677T substitution in the methylenetetrahydrofolate reductase gene in Manitoba [Letter]. Clin Genet 2000; 58: $406-408$.

16 Frosst P, Blom HJ, Milos R et al: A candidate genetic risk factor for vascular disease: a common mutation in methylenetetrahydrofolate reductase [Letter]. Nature Genet 1995; 10: 111-113.

\section{Reply to 'MTHFR C677T and A1298C polymorphisms and mutated sequences occurring in cis'}

European Journal of Human Genetics (2002) 10, 579-582. doi:10.1038/sj.ejhg.5200863

Isotalo and Donnelly comment in their letter (2002, in this issue) on the conflicting reports regarding the prevalence of MTHFR 677T and 1298C alleles occurring in cis.
In our study we did not find any triple or quadruple MTHFR mutation combinations either in the spontaneous abortion group $(n=80)$ or in the control group which 